City University of New York (CUNY) CUNY Academic Works

\title{
A stochastic mesoscale model of transiently networked fluids.
}

Lin Zhou

CUNY New York City College of Technology

L. Pamela Cook

University of Delaware

\section{How does access to this work benefit you? Let us know!}

More information about this work at: https://academicworks.cuny.edu/ny_pubs/552

Discover additional works at: https://academicworks.cuny.edu

This work is made publicly available by the City University of New York (CUNY).

Contact: AcademicWorks@cuny.edu 
Zhou, L., \& Cook, L. P. (2017). A stochastic mesoscale model of transiently networked fluids. Journal of NonNewtonian Fluid Mechanics, 247, 78-89. https://doi.org/10.1016/j.jnnfm.2017.06.004

(c) 2017. This manuscript version is made available under the CC-BY-NC-ND 4.0 license http:// creativecommons.org/licenses/by-nc-nd/4.0/

\title{
A STOCHASTIC MESOSCALE MODEL OF TRANSIENTLY NETWORKED FLUIDS
}

\author{
LIN ZHOU \\ Department of Mathematics, New York City College of Technology, CUNY \\ L.PAMELA COOK \\ Department of Mathematical Sciences, University of Delaware
}

\begin{abstract}
Macroscale models of physically networked fluids suffer from the inability to connect directly to mesoscale processes due to nonlinearity and thus the need for closure approximations. In this paper physically networked complex fluids are modeled stochastically at the mesoscale, thus avoiding the need for closure assumptions. In the modeling and simulations the network consists of linear chains of Hookean bead-spring dumbbells. The linear chains break and reform stochastically subject to prescribed local attractive potentials. In the simulations the topology of the network is tracked allowing for quantification of the distribution of chain lengths and of individual dumbbell stretch. This formulation allows for breakage and recombination associated with local stress. Model predictions of the distributions of chain lengths and of dumbbell stretch both in equilibrium and in steady shearing are presented. As well, shear thickening and shear thinning regimes in steady shearing and relaxation after step strain are examined.
\end{abstract}

\section{INTRODUCTION}

Many complex fluids consist of a network of "chains" at the mesoscale. The network connections may be permanent or transient (as entanglements in entangled polymers, temporary connections of telechelic polymers, or as in the breaking and reforming micellar worms in surfactant solutions). The macroscale properties of these fluids depend not only on the macroscale environment such as temperature and concentration, but also on local (at the mesoscale) properties such as stretch and orientation of chain elements, local flow deformation and local concentration of surfactant and salt, all of which may vary due to demixing and flow inhomogeneities. These local properties may affect the local attractive force of a chain end in physically associating networks and, in the case of wormlike micelles, the energy needed to create new endcaps. The connections, entanglements, or crosslinks cause the fluid to evidence elastic properties; if the connections, entanglements or crosslinks are temporary the fluid may evidence elastic properties on a fast (short) time scale but 
solely viscous fluid properties on a slow (long) time scale. In these fluids local changes at the mesocale can have global effects at the macroscale.

Wormlike micelles are a particular type of transiently networked fluids. In a watery solvent the amphiphilic surfactant molecules self-assemble to protect their tails from the water. Under certain conditions the resultant "structure" is a self-assembled long floppy worm in which the heads (hydrophilic) form the outer shell of the cylindrical structure, and the tails (hydrophobic) are "hidden" inside [1,2]. These worms continuously break and reform in addition to, similar to long polymer strands, entangling. In flow, the breakage rate appears to be enhanced from that at equilibrium which is thought to be due to the local stretch of the worms which, when stretched, carry more stress. Experiments show that in shear rate controlled flow conditions the breakage/reforming and the entanglement can lead to shear banding $[3,4,5]$. The resultant shear bands are thought to consist of regions of high shear rate in which primarily only short (broken) aligned worms exist and regions of low shear rate in which a roughly equilibrium distribution of short and long worms exist. The shear banding is believed to be a consequence of an instability (a nonmonotonicity) in the rheometric flow curve (stress versus shear-rate at a controlled shear rate). This same nonmonotonicity is seen in surfactant-telechelic networked systems [6].

The inhomogeneous flow of wormlike micellar solutions has been investigated using macroscopic models. Examples include the two species breaking and reforming VCM model $[7,8,9]$ which has predicted good agreement with experimental results for $\mathrm{CPyCl}$ in $\mathrm{NaSal}$ in both shear and extensional flows and the GCB model [10]. Both models incorporate the kinetics of the worms, the breakage and reforming, in a simplified discrete (two species) way. The GCB model, a thermodynamically consistent variant of the VCM model, has the advantage that breakage/reforming rates are determined solely by the stress, whereas the VCM model breakage rates are determined by both the stress and the shear rate. Other models include the single species phenomenological Johnson-Segalman model [11] which predicts shear banding in steady shearing flow, but predictions of which do not agree with experimental results under step strain nor under extension; and the Giesekus model which is a Maxwell model with the addition that it allows for the friction coefficient to depend on the polymer or worm orientation through the macroscale stress $[12,13]$. In any of these models, even though derived from a mesoscale framework, it is difficult to ascertain how the models should be generalized to cover changes in type of surfactant, concentration, salinity, or temperature of the mixture. In addition to the demixing/shearbanding properties evidenced by wormlike micellar solutions at the macroscale, experiments show that as conditions change (concentration, salinity, temperature) the relaxation after a small step strain can be much slower (power-law or stretched exponential) [14] than the typical exponential decay evidenced by the VCM and GCB models which are nonlinear two mode variants of the upper convected Maxwell model and hence show exponential relaxation in linear theory, small step strain. Similar stretched exponential relaxation has been observed in surfactant-telechelic systems $[6,15]$. Closer investigations and a broader understanding of what the energy functionals and parameter regimes are that lead to this slow relaxation 
are needed. For these reasons we re-explore the modeling from the mesoscopic level, and look for direct, not ensemble or spatially averaged, stochastic mesoscopic models and simulations that may have the ability to cover the behavior of the networked mixtures under a range of concentration, temperature, and salt variations.

Direct mesoscale stochastic models of wormlike micellar solutions have been formulated and simulated in the linear regime including both reptative and breakage effects by [16] and more recently with added mode mechanisms in [17]. In [17] the theory of [16] was extended to consider more physics including the effects of chain length fluctuations (CLFs), constraint release (CR), Rouse modes, and semi-flexibility (bending modes). The authors were able to transfer their time domain results to the high frequency domain thus allowing for comparison with data over the full frequency range. While this is a highly detailed and valuable study, it is limited to the linear response regime. $[18,19]$ proposed an extension of [16] to the nonlinear regime in the form of an integral model driven by shear induced tube alignment. The goal in this paper is to consider a tractable nonlinear model in which the stress dependence of the breakage/reforming drives the model. Initially we do not consider the full range of physics as in [17], but we do consider variations in the functional dependence of the local breakage/reforming rates. The mesoscale models considered in this paper are closer to the macroscale GCB model than the VCM model because in the latter model the breakage rate depended both on the averaged stress and on the shear rate, whereas in this work the breakage/reforming depends only on the local stress.

Stochastic mesoscale two mode transient elastic network models have been formulated and simulated primarily in the context of associating polymers [20, 21, 22]. In [20] the spatial location of each bead was tracked as well as its connections to other beads so that a physical network was realized. In [21] bead connections were not tracked. Rather, beads were determined stochastically to be in one of two states, either attached or detached with attached beads being identified by having a high friction coefficient, the detached beads a comparatively low friction coefficient in the equations of motion. The high friction coefficient was intended to simulate the effect of the impeded motion due to the network attachments. The stochastic breakage process was formulated assuming an attractive parabolic potential well for a junction, which means the resultant breakage depended on the spring stretch, that is the local stress. The recombination rate was assumed to increase linearly with the spring stretch. Both Hookean and FENE dumbbells were considered in that work. In [22] the analysis was extended to consider bead-spring chains of a fixed number of beads. As before each bead was labelled via a stochastic rule as either attached (to a bead of another chain) or detached and given a corresponding high or low friction coefficient in the equation of motion. Other authors have considered ensemble averaged mesoscale and macroscale models in which two distinct species are identified, one in which dumbbells have both beads attached to the network, the other in which dumbbells have one free bead $[23,24]$. In [24] the ensemble averaged and macroscale breakage rates were derived from a mesoscale detachment involving an energy balance. In $[23,24]$ beads were assumed to attach at a rate that increased linearly with chain stretch and attached beads were assumed 
to move affinely, thus an extreme of [21] in which the friction of the attached bead is infinite.

In this paper the "worm" is modeled directly as connected bead-spring dumbbell units as in [20], and the full topology of the system, that is the spatial location of each bead and spring, and the spatial extension and connections of each spring/dumbbell are tracked. In the model, dumbbell units are connected through sticky nodes [20] to form long chains and the chains break at a node position if a sticky node dies. Different from [20], in which the breakage and reforming parameters remain constant, in our work we consider various situations including that the network breakage and reforming have local dependence on stress such that highly elongated connecting strands which carry enhanced stress are more apt to detach and less apt to attach. The model resembles a polydisperse ensemble of Rouse chains with an additional relaxation mechanism due to the continual breakage and reforming of the chains. This modeling approach avoids the need for averaging assumptions in order to obtain closure at the macroscale level, avoids having local breakage rates depend on a spatial average of end to end stretch as in the macroscale models, and allows us to track the chain lengths. Using this mesoscale model has some drawbacks, for example the persistence length (stiffness) of the worm segments is not considered and reptation is lumped into a spring stiffness. However, this modeling effort does elucidate the impact of breakage and reforming from the mesoscale to the macroscale and allows us to track the worm topology, a first step in understanding the mesoscale effects on the macroscale dynamics.

The paper is organized in 8 sections. Section 1 is the introduction. In Sections 2 and 3 we introduce relevant background material and our model development. In Section 4, we briefly describe the numerical scheme and the parameters used in the simulations. Sections 5, 6 and 7 each present and discuss the results of the model simulation in equilibrium, step strain and shear rate controlled flow, respectively. Section 8 concludes the paper.

\section{BACKGROUND}

Modeling of complex fluids often begins at a "coarse-grained" level, for example polymer or wormlike micellar mixtures are often modeled using bead-spring dumbbells as an approximation to a full flexible chain. In the Rouse model, Hookean bead-spring dumbbells are connected so that $N_{d}$ dumbbells constitute a chain. In these bead-spring models, the starting point is the Langevin equation which describes the force balance on the beads in the solvent incorporating viscous drag, the (Hookean) elastic spring force and the Brownian force. When the bead of a dumbbell is assumed inertialess, the dimensionless Langevin equation for the position of the $i^{t h}$ bead of a single dumbbell at time $t+\Delta t$ is given as [25]:

$$
\mathbf{r}_{i}(t+\Delta t)=\mathbf{r}_{i}(t)+\left(\mathbf{v}_{\mathbf{0}}+\boldsymbol{\kappa} \cdot \mathbf{r}_{i}(t)\right) \Delta t+\frac{1}{4} \mathbf{Q}_{i j} \Delta t+\Delta \mathbf{w} .
$$

Here $i, j=1,2$ and $i \neq j$. In the equation, time is nondimensionalized by the relaxation time of a single dumbbell $\lambda=\zeta / 4 H$, with $\zeta$ being the hydrodynamic drag coefficient and $H$ being the (Hookean) spring constant; length is scaled by $\sqrt{k T / H}$, the length of a free 
dumbbell in equilibrium, in which $k$ is the Boltzmann constant and $T$ the temperature. $\mathbf{v}_{0}+\boldsymbol{\kappa} \cdot \mathbf{r}$ is the imposed homogeneous shear flow field at the bead position $\mathbf{r}$ and $\boldsymbol{\kappa}=(\boldsymbol{\nabla} \mathbf{v})^{t}$, the transpose of the velocity gradient. The stochastic increment $\Delta \mathbf{w}$ follows a Gaussian distribution with zero mean and dimensionless variance $\sigma^{2}=\Delta t / 2$ (a Wiener process). Here $\mathbf{Q}_{i j}=\mathbf{r}_{j}-\mathbf{r}_{i}$.

For this linear equation/process it is known $[26,25]$ that the ensemble motion is governed by the mean field, the Fokker-Planck equation for the distribution of the number density of dumbbells $\psi$ in configuration space $\mathbf{Q}$, the connector vectors of the dumbbells, namely:

$$
\psi_{, t}(\mathbf{Q}, t)=-\left\{\boldsymbol{\nabla}_{\mathbf{Q}} \cdot\left(\boldsymbol{\kappa} \cdot \mathbf{Q}-\frac{1}{2} \boldsymbol{\nabla}_{\mathbf{Q}} \ln \psi-\frac{1}{2} \mathbf{Q}\right) \psi\right\} .
$$

The macroscopic stress $\boldsymbol{\sigma}$ is the second moment of $\psi[26], \boldsymbol{\sigma}=\int \mathbf{Q} \mathbf{Q} \psi(\mathbf{Q}, t) d \mathbf{Q}$. For this linear model the integrations can be carried out explicitly to give:

$$
\boldsymbol{\sigma}_{(1)}+\boldsymbol{\sigma}-\mathbf{I}=0
$$

which is the upper convected Maxwell (UCM) model with the upper convected derivative given by $\boldsymbol{\sigma}_{(1)}=\boldsymbol{\sigma}_{, t}-(\boldsymbol{\nabla} \mathbf{v})^{t} \cdot \boldsymbol{\sigma}-\boldsymbol{\sigma} \cdot \boldsymbol{\nabla} \mathbf{v}$.

Thus to "solve" the problem and find the resultant stress of the system one can either simulate the bead motions at the mesoscale (thus tracking each dumbbell using equation (1)), then add the dyadic product of the connector vectors to get the macroscopic stress, $\boldsymbol{\sigma}=\sum \mathbf{Q}_{i} \mathbf{Q}_{j} / N_{t}$ in which $N_{t}$ is the total number of dumbbells; or solve the Fokker-Planck partial differential equation (2) in configuration space, then take the second moment; or solve the (quasi-linear) upper convected Maxwell equation (3) directly, all with appropriate initial and boundary conditions. Similar solution choices hold for a Rouse chain of $N_{d}$ dumbbells [25]. However, the occurrence of nonlinearities in the model: nonlinear spring force and nonlinear breakage or reforming of chains; possible non Gaussian stochastic processes; aging; and/or inhomogeneities in the flow field complicate or make these equivalent options impossible.

\section{Model Development}

We follow the paper of [20] by considering a connected network of dumbbells and sticky nodes in which the topology of the network is tracked during the simulation. Fig. 1 shows a schematic diagram of the simulation. Every strand is modeled as a linear elastic beadspring dumbbell. A bead of one dumbbell can attach to beads of other dumbbells through sticky nodes. A node with attached beads is a junction. A sticky node may have up to $f$ beads attached to it, but each bead can stick to only one node. Here $f$ is defined as the functionality of the node. In the simulations in this paper we will consider the case when the functionality of the node is 2 , that is, $f=2$. 


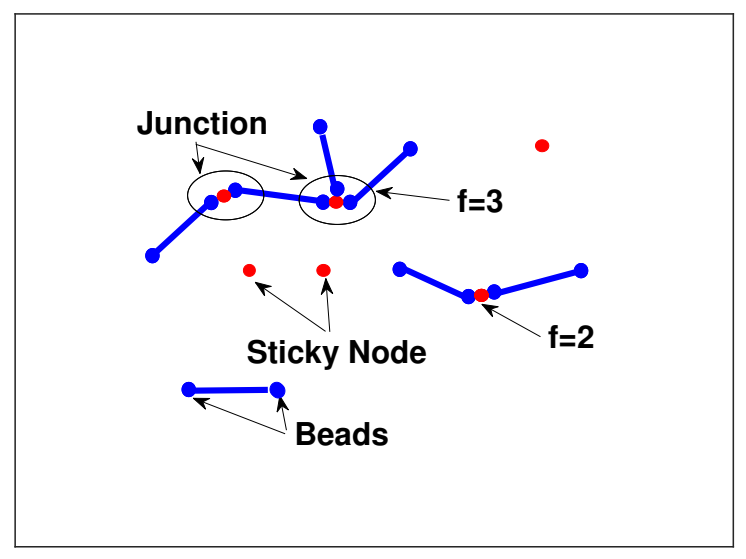

FIGURE 1. Schematic of a transient network showing the sticky nodes (in red) and junctions of functionality $f$ being 2 and 3 .

Following the same model formulation as [20], the position of each inertialess bead at time $t+\Delta t$ is updated through the following dimensionless equation:

$$
\mathbf{r}(t+\Delta t)=\mathbf{r}(t)+\left(\mathbf{v}_{\mathbf{0}}+\boldsymbol{\kappa} \cdot \mathbf{r}(t)\right) \Delta t+\frac{1}{4} \mathbf{Q} \Delta t+\Delta \mathbf{w}
$$

Here, as in (1), the length is scaled by the equilibrium length of a free dumbbell, $\sqrt{k T / H}$, time by the relaxation time of a single dumbbell, $\zeta / 4 H$. To describe the motion of a junction, the same equation as (4) is used except that the forces are the sum of the forces on each bead connected to the junction divided by the total number of beads of the junction. Nodes which have no beads attached jump randomly at each time step in space.

Since each node can connect up to 2 beads, this process allows linear chains containing varying numbers of dumbbells to be formed. The breakage and reforming of chains are controlled by the sticky nodes. When a node dies, the junction it forms vanishes, and the node reappears as a free node at a randomly determined location. If the mean lifetime of a node is $\tau$, then $1 / \tau$ is the mean death rate. Following the Poisson distribution, the probability that a node does not die in the time interval $\Delta t$ is $e^{-\Delta t / \tau}$, so the probability of breakage of a junction in each time step is $1-e^{-\Delta t / \tau}[20]$. For the attachment of a node and a bead we assume that, if a bead is within a prescribed distance $d$ of a node which has a vacancy, the bead attaches to the node [20]. Here $d$ is the capture radius.

Our first simulations are carried out with the mean node lifetime $\tau$ and the capture radius $d$ being constant, similar to [20]. In our later simulations the node lifetimes $\tau$ and the capture radius $d$ will depend on the local stretch. Two scenarios are considered for this 
local stress dependent attachment/detachment process.

In the first scenario the beads attached to a node are considered to be residing in an attractive parabolic potential well as in $[21,22]$. In dimensionless variables the attractive potential is give by $U=-U_{0}\left(1-\frac{r^{2}}{d_{0}^{2}}\right)$ for $r<d_{0}$ and $U=0$ otherwise. Here $U_{0}$ is the depth of the potential well and $d_{0}$ is the width. The bead is able to free itself from the node if either the node dies through thermal variations, or if the force exerted on the attached bead by its spring exceeds the attractive force of the node. That balance occurs when $Q=\frac{d U}{d r}$ where $Q=(\mathbf{Q} \cdot \mathbf{Q})^{1 / 2}$. Solving that relationship for $r$, then substituting that value back into the potential gives $U=-U_{0}\left(1-\frac{d_{0}^{2} Q^{2}}{4 U_{0}^{2}}\right)$. The mean node lifetime is then

$$
\tau \propto e^{-U} \propto e^{U_{0}} e^{-U_{0}\left(\frac{d_{0} Q}{2 U_{0}}\right)^{2}}=\tau_{0}\left(U_{0}\right) e^{-U_{0}\left(\frac{d_{0} Q}{2 U_{0}}\right)^{2}}
$$

where $\tau_{0}\left(U_{0}\right)$ is the lifetime of the node if there is no dependence on $Q$.

Similarly, the attachment of a bead to a node depends on the distance between the bead and the node as well as on the balance of the node attractive force and the spring force on the bead. Just as the force exerted by the stretched spring may support the detachment process it may also impede the capture/attachment process. A bead can attach to the node only if it is within a distance $d_{0}$ of the node and also the spring force is not strong enough to counteract the maximum attractive force of the node. Balancing the spring force of the bead and the maximum attractive force, the attachment can occur when the distance of the bead to the node is less than $\min \left(d_{0}, d_{F}(Q)\right)$, where $d_{F}(Q)=2 U_{0} / Q$, that is, the capture radius is:

$$
d=\min \left(d_{0}, d_{F}(Q)\right)=\min \left(d_{0}, \frac{2 U_{0}}{Q}\right) .
$$

In the second scenario detachment occurs if the retractive energy of the bead's spring $\int_{Q}^{Q-d_{0}} Q d Q$ is greater than or equal to the (constant) attractive energy of the node $U_{0}$ as in [24]. In this case the mean lifetime of the node, $\tau$, is proportional to $e^{U_{0}+\int_{Q}^{Q-d_{0}} Q d Q}$, or

$$
\tau \propto e^{U_{0}} e^{-d_{0} Q+\frac{d_{0}^{2}}{2}}=\tau_{0}\left(U_{0}\right) e^{-d_{0} Q+\frac{d_{0}^{2}}{2}} .
$$

Similarly, for attachment to occur the bead must be within a distance $d_{0}$ of the node and the node attractive energy must be strong enough to stretch the spring, that is, $U_{0} \geq$ $\int_{Q}^{Q+d_{0}} Q d Q$. Thus for attachment the distance of the bead from the node must be less than or equal to $\min \left(d_{0}, d_{P}(Q)\right)$ where $d_{P}=-Q+\sqrt{Q^{2}+2 U_{0}}$, that is, the capture radius is:

$$
d=\min \left(d_{0}, d_{P}(Q)\right)=\min \left(d_{0},-Q+\sqrt{Q^{2}+2 U_{0}}\right) .
$$


In the above two scenarios both the breakage and the reforming are consistent with the kinetics as opposed to an ad hoc reforming assumption as in previous works [21, 22, 24].

\section{Numerical Scheme}

The simulations are carried out in a box of dimensions of 20 by 5 by 5 (in $\sqrt{k T / H}$ units) as in [20], with 2,000 dumbbells and 1,000 or 2,000 sticky nodes. In the case of 2,000 nodes, there are an average of 4 dumbbells per unit cube, and 2 beads for each node. In the case of 1,000 nodes there are an average of 4 beads for each node. Since each node is only allowed to attract two beads, at this node level there are a number of beads left unattached. The number of sticky nodes affects the degree of networking. With 2,000 nodes the dumbbells can form one single long linear chain of all dumbbells, while for 1,000 nodes the maximum length chain would utilize only half the dumbbells.

Initially, nodes and dumbbells are generated at random positions from a linear distribution in each direction. Nodes and beads are numbered. The positions of the nodes and beads and their connection information are recorded at each time step, so the topology of the network is tracked. At each time step, we investigate the nodes one by one in the numerical order. If a node dies, it releases all the beads that are connected to it and the node is reborn in a location determined randomly. The released beads move to a new position following the governing equations. If a node is still alive, then it (together with its attached beads) moves to a new position following the governing equations. We then investigate if there are any beads within the capture radius of the node. If so, then that bead attaches to the node from the closest first, up to the capacity of the node. At each time step, the remaining beads move to a new position determined by the governing equations. Periodic boundary conditions are used in all three $(x, y, z)$ spatial directions in equilibrium. When the system is subjected to shearing flow, Lees-Edwards boundary conditions [27] are used in the flow direction. In the simulations, a typical time step is 0.01 [20]. At equilibrium, for the range of parameters studied in this paper, the stress matrix $\boldsymbol{\sigma}$ is the identity.

The nondimensionalized models involve several parameters: the number of nodes $N$, the capture radius $d$, and the mean node lifetime $\tau$. When breakage and reforming are inde-

pendent of the stretch of dumbbells, $\tau$ and $d$ are constant. When breakage and reforming are dependent on the stretch of dumbbells, there are two one more parameters $U_{0}$ and $d_{0}$. In this case, $\tau$ and $d$ both depend on $U_{0}$ and $d_{0}$ with the functional forms provided in Eqs. (5)-(8) for the two different scenarios. In selecting constant values for these parameters we follow the work of $[20,21]: N=1000$ or $2000 ; U_{0}$ varies from 10 to $12 ; d_{0}=0.175$ or 0.3 ; when $\tau$ and $d$ are constant, $\tau=0.25 \sim 10$ and $d=0.175,0.3$. 


\section{Equilibrium Results}

Initially all dumbbells and nodes are free (not connected). Then, the system is allowed to equilibrate. The shear stress and normal stresses as functions of time, as well as the distribution of dumbbell end to end distance $Q$ at both the initial and equilibrated status are shown in Fig. 2 and 3 for two different sets of constant parameters: the mean lifetime of the nodes $\tau$, the capture radius $d$, and the number of nodes $N$. In Fig. $2, \tau=1$, $d=0.175$ and $N=1,000$; in Fig. $3, \tau=10, d=0.3$ and $N=2,000$. In both figures, the time evolution of the stresses indicates that the shear stress equilibrates faster than the normal stresses, and the normal stress in the larger direction of the simulation box (the flow direction $x$ ) takes longer time to equilibrate than those in the two shorter directions. The reason for this is that the bead locations are initially distributed randomly in the box. If the dimension is larger in one direction, then beads are spaced further apart initially (which means higher stretches $\mathbf{Q}$ ) in that direction. Hence, it takes a longer time for the system to relax in that direction. Comparing the two figures, the time for the normal stresses to equilibrate increases as $\tau, d$ and $N$ increase. In Fig. 2 and Fig. 3, the initial distribution of the magnitude of the stretch of each dumbbell $Q$ is highly spread. After equilibration, the distribution of $Q$ follows the equilibrium stretch distribution of single dumbbell mixtures $\frac{4 \pi Q^{2}}{(2 \pi)^{3 / 2}} \exp \left(-\frac{Q^{2}}{2}\right)[26]$.
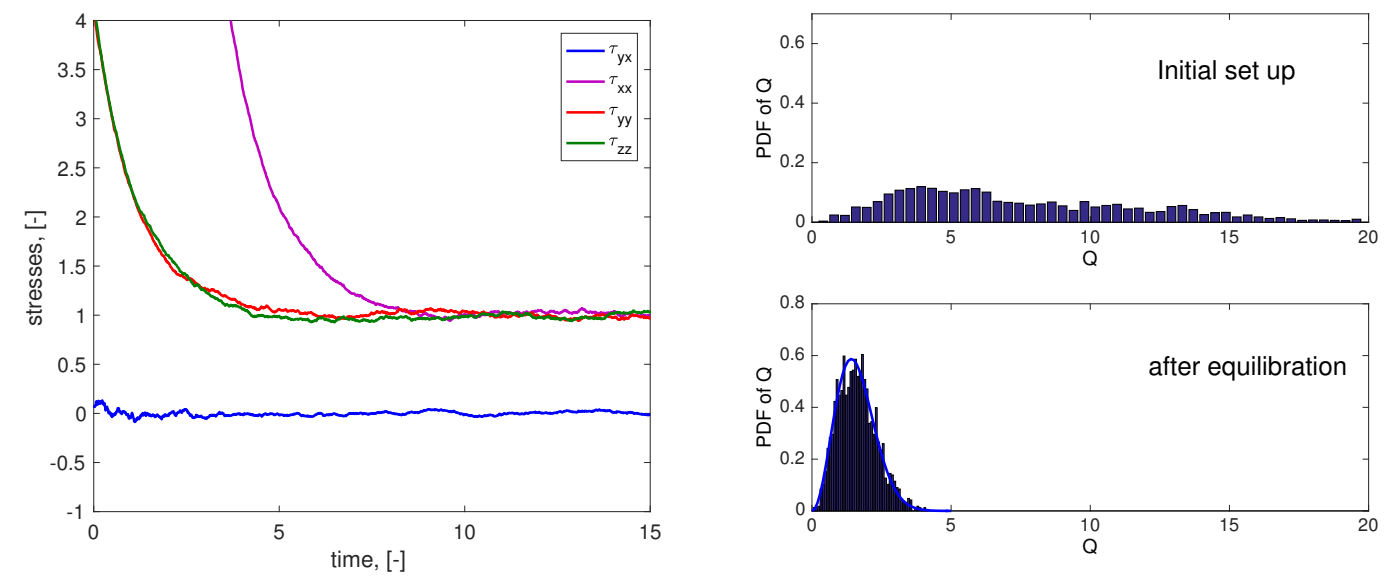

Figure 2. The left figure shows the evolution of normal and shear stresses as the system equilibrates. After equilibration, normal stresses equal 1 and the shear stress is 0 . The top right figure shows the distribution of the magnitude of the stretch of each dumbbell $Q$ in initially prescribed status. The bottom right figure shows the same $Q$ after the equilibration. The blue solid line in the bottom right figure represents the function $\frac{4 \pi Q^{2}}{(2 \pi)^{3 / 2}} \exp \left(-\frac{Q^{2}}{2}\right)$. In the figure the parameters are $\tau=1, d=0.175, N=1,000$. 

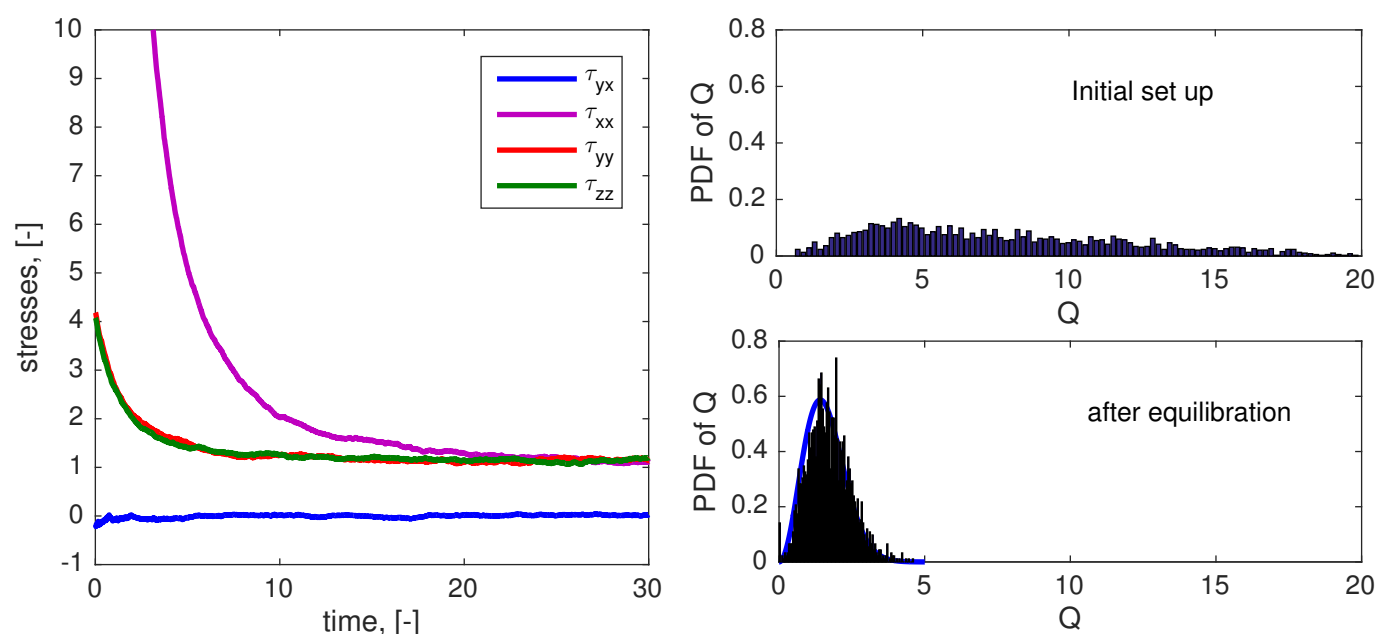

FiguRE 3. The left figure shows the evolution of normal and shear stresses as the system equilibrates. The right figures show the distribution of the magnitude of the stretch of each dumbbell $Q$ in initially prescribed status (top) and after the equilibration (bottom). The parameters are $\tau=10, d=0.3, N=2,000$.

Cates, in a model of continuous breakage and reforming of wormlike micelles, the breakage with equal probability at any point along the length of the worm, constructed an ordinary differential equation in time governing the number density of worms of a given length $\mathcal{N}(L)$ [16]. Cates showed that the equilibrium distribution of lengths was exponential, $\mathcal{N}(L) \sim e^{-L / \bar{L}}$, where $\bar{L}$ is the mean length of the chains of the mixture [16]. In our simulation, because we track the topology of the network, we can count the number $L$ of dumbbell units per chain in equilibrium and verify Cate's theory for our discrete version. Fig. 4 shows the number density of chains containing $L$ dumbbells at equilibrium in the simulation (discrete symbols in the figure) for various parameter values and their exponential fittings (solid lines in the figure) by the least square method. In all cases, in agreement with Cates' results, the number density of chains with length $L, \mathcal{N}(L)$, shows exponential dependence. In the right figure of Fig. 4, for the higher mean node lifetimes, the length distribution becomes fairly flat, hence the curves are plotted in semi-log coordinates in the inset for a clearer presentation to show the exponential dependence.

Fig. 4(left) exhibits the number density functions for $N=1,000, d=0.175$ and various values of $\tau$. Fig. 4(right) shows the same functions for $N=2,000, d=0.3$ and several values of $\tau$. The figures show that the distribution is dependent on the value of the mean lifetime $\tau$ with the distribution flattening (more longer chains, that is larger $\bar{L}$ ) for larger $\tau$, and highly dependent on the value of $N$, the number of nodes. For $N=1,000$, the fitted mean length for all cases shown is close to 1 . For $N=2,000$ and $\tau=10$ the distribution is fairly flat with a much longer fitted mean length, close to 10 . The chains are 

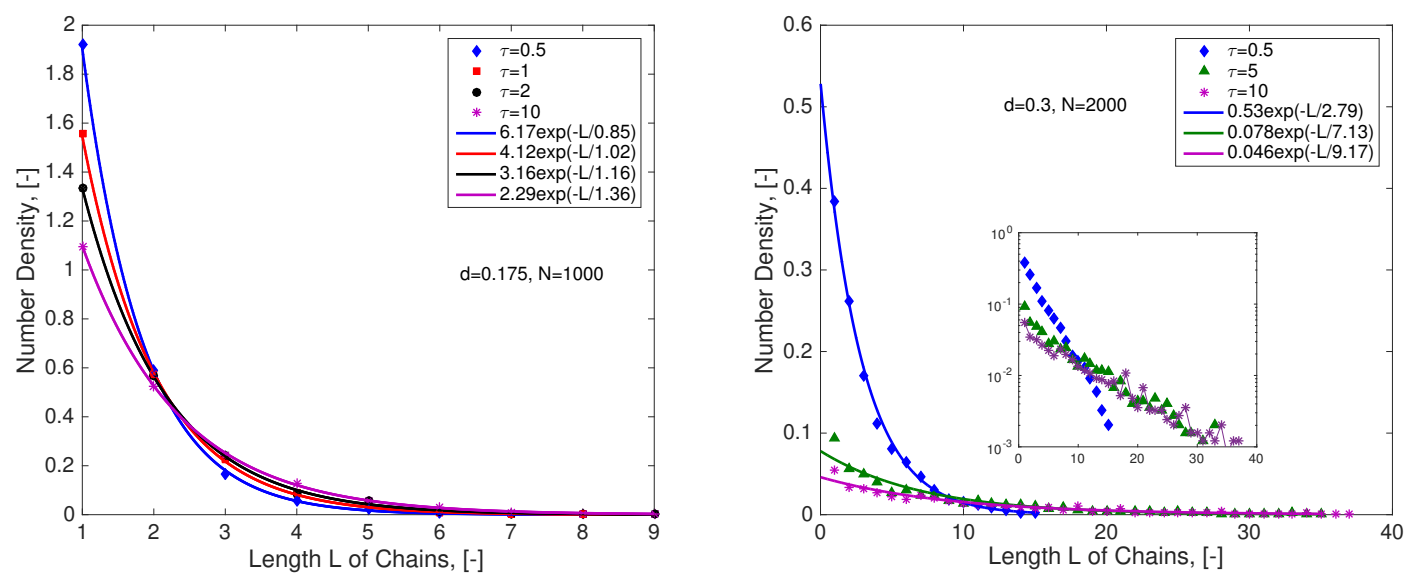

FiguRE 4. Number density of chains of $L$ connected dumbbell units at equilibrium. Left: the parameters of the curves are $d=0.175, N=1,000$ and $\tau=0.5,1$, 2, 10. Right: the parameters are $d=0.3, N=2,000$ and $\tau=0.5,5,10$. The inset of the right figure is a semi-log plot.

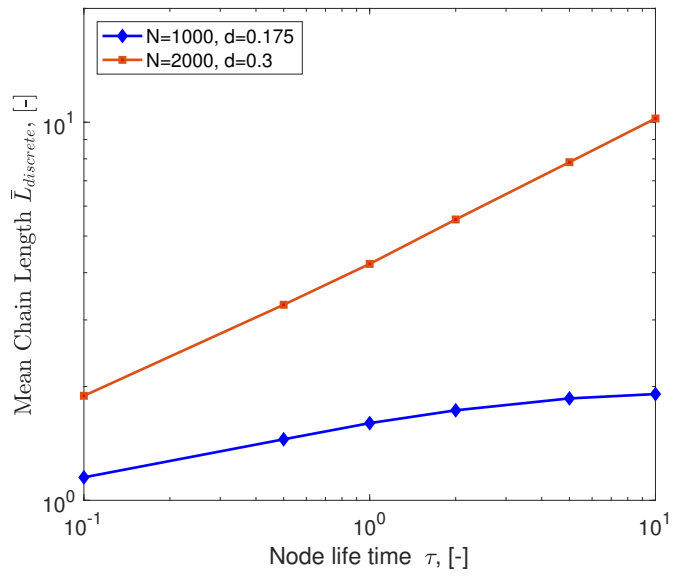

FIGURE 5. The discrete mean chain length as a function of mean node lifetime $\tau$ for two cases: $N=1000, d=0.175$, and $N=2000, d=0.3$.

composed of many more dumbbell units, on average, for larger $N$. An increase in $N$, the number of nodes in the simulation, effectively is an increase in the allowed connectivity of the network. It is important to realize that our model is discrete with the smallest length unit being $L=1$ so that while we did fit our results of the distribution of $L$ to continuous (exponential) curves in Fig. 4, the discrete mean length of the chains differs from that for the continuous assumption. The discrete mean length of the chain, $\bar{L}_{\text {discrete }}$, is defined as 
the ratio of the total number of dumbbells to the total number of chains formed in the system. The dependence of the discrete mean chain length $\bar{L}_{\text {discrete }}$ on $\tau$ in Fig. 4 is summarized in Fig. 5. When $N=1000$, the maximum main chain length is 2 , no matter what the configuration, so $\bar{L}_{\text {discrete }}$ approaches 2 as $\tau$ increases. When $N=2000$, the maximum mean chain length can be 2000, thus allowing $\bar{L}_{\text {discrete }}$ to continue increasing as $\tau$ increases. The figure shows that this dependence is $\bar{L}_{\text {discrete }} \sim \tau^{1 / 3}$.

\section{Relaxation AFTER STEP STRAin}

To investigate network relaxation dynamics we consider the behavior of the model after the imposition of a step strain. A shear rate of 100 is imposed over one time step $\Delta t=0.01$, a net strain of 1 . Thereafter the shear rate reverts to zero and the shear stress relaxation dynamics are tracked. In the following figures (Figs. 6, 7, 8), curves shown are an average of 10 simulations. The results in the section are for constant mean node lifetime $\tau$ and constant capture radius $d$.
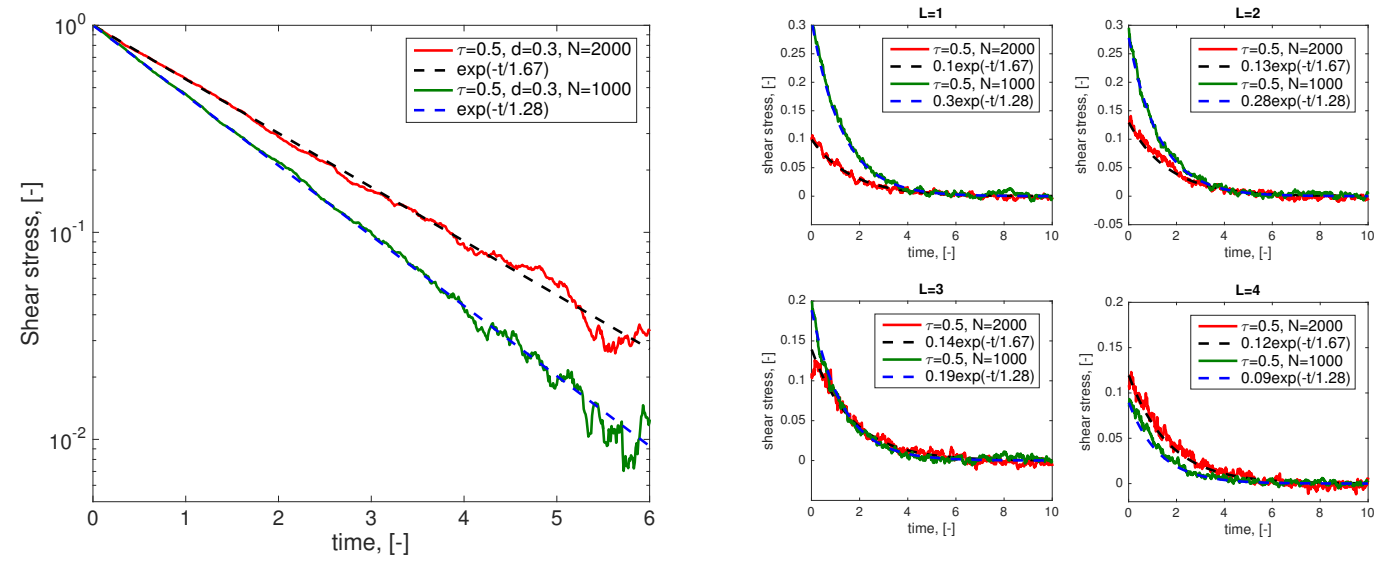

FiguRE 6. The left figure shows the step strain relaxation curves for different node numbers $N$, with $d=0.3$ and $\tau=0.5$. The right figures are the stress relaxation in time with the contribution from each of the chains of $L=1,2,3$ and 4 dumbbells reported individually.

Fig. 6 shows the shear stress relaxation for the number of nodes $N=1,000$ and 2,000 for a capture radius $d=0.3$ and a mean node lifetime $\tau=0.5$. For this short mean node lifetime, breakage dominates the relaxation process and both relaxations are exponential. The relaxation time of the network, $\lambda_{n w}$, is $\lambda_{n w}=1.28$ and 1.67 respectively for the $N=1,000$ and $N=2,000$ cases. To investigate the contribution to the total relaxation from each length group, we checked the relaxation behavior of chains of $L=1,2,3$, and 4 respectively and plotted them on the right figure of Fig. 6. Contributions from even longer chains are 

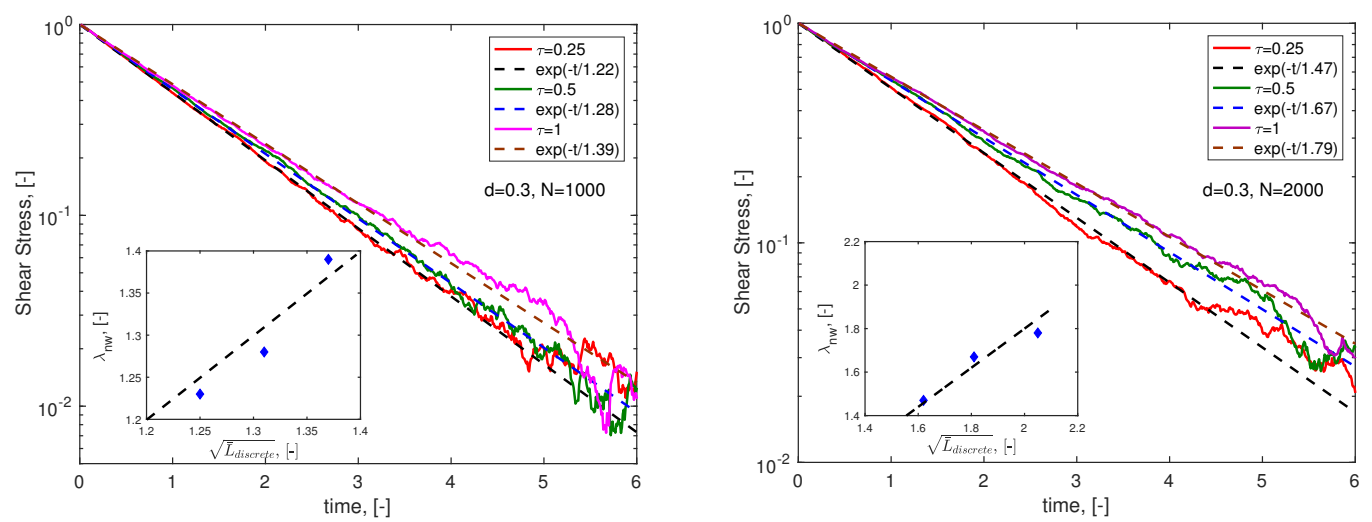

FIgURE 7. Step strain relaxation for node number $N=1,000$ (left) and node number $N=2,000$ (right) with various mean node lifetimes $\tau=0.25,0.5,1$ and $d=0.3$. The insets of each figure represent the relaxation time as a function of the mean length $\left(\bar{L}_{\text {discrete }}\right)^{1 / 2}$ of the system. The dashed line in the inset of the left figure has slope 1 , and the dashed line in the inset of the right figure has slope 0.9 .

not presented here. Note that each group shows the same relaxation behavior (relaxation time) as the system. Note that as the number of nodes increases, the relaxation time of the network as well as the chains of a given length increases. The relaxation of the system is the sum of the relaxations of different length groups each with the same exponential function.

Fig. 7 shows the relaxation after step strain for the number of nodes $N=1,000$ (left) and $N=2,000$ (right) for three different mean node lifetimes $\tau=0.25,0.5,1$. These mean node lifetimes are all shorter than or equal to the relaxation time of a single dumbbell (which is 1 in this scaling). In this range the relaxation dynamics are all single exponential $e^{-t / \lambda_{n w}}$ with relaxation times $\lambda_{n w}$ increasing as $\tau$ increases. Notably, as anticipated, as $\tau$ goes to 0 the relaxation time approaches 1 . The insets in Fig. 7 show that for these short mean node lifetimes the relaxation time of the ensemble is roughly a linear function of the square root of the mean length of the ensemble $\left(\bar{L}_{\text {discrete }}\right)^{1 / 2}$, that is, $\lambda_{n w}=a(N)\left(\bar{L}_{\text {discrete }}\right)^{1 / 2}$ where the linear coefficient $a(N)=1$ or 0.9 for $N=1,000$ or 2,000 , respectively.

Fig. 8 shows the step strain relaxation for a larger mean node lifetime namely $\tau=5$, for the same values of $d$ and $N$ as in Fig. 6. For this larger constant mean node lifetime the relaxation is no longer single exponential but instead shows exponential behavior at short times and is best fit with a stretched exponential for larger times $(t>1)$. For $N=1,000$ the contribution to the stress from the shorter chains, $L=1,2,3$ and 4 is plotted on the right of Fig. 8. For this mean node lifetime and $N=1,000$ the relaxation of each length chain group is single exponential, the relaxation times for $L=1,2,3,4$ are, respectively, 

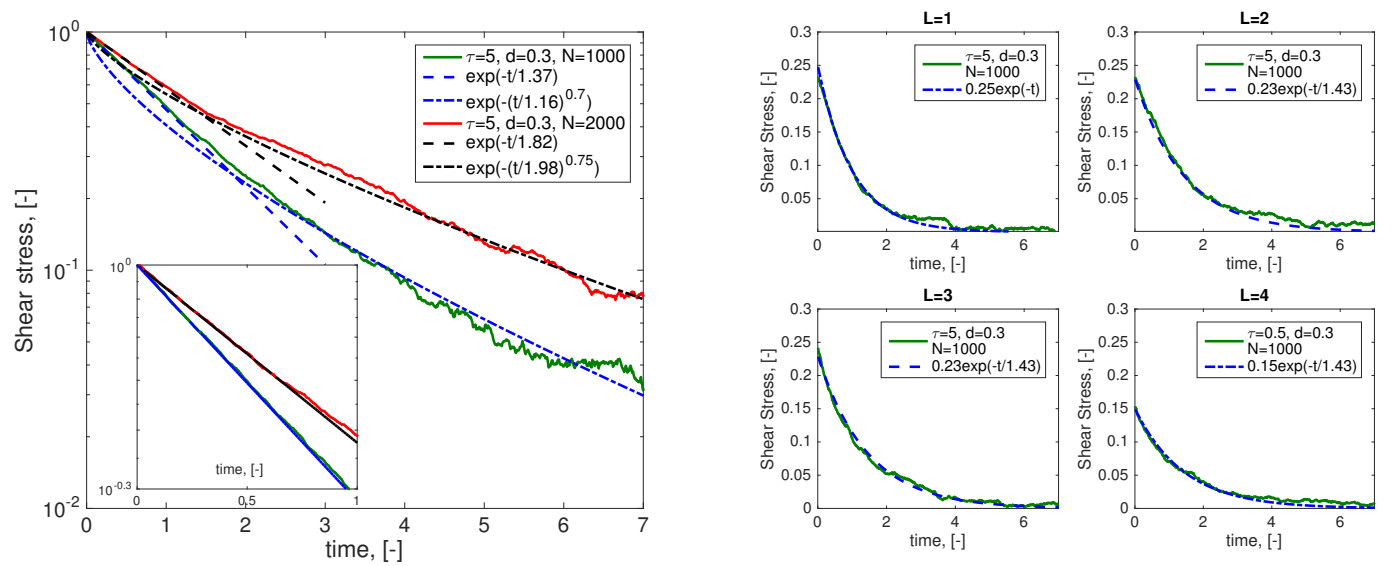

FiguRE 8. The left figure shows step strain relaxation for $\tau=5, d=0.3$ and $N=1,000$ or 2,000 , respectively. The inset is the blow up of the behavior up to $t=1$. The right figure shows the contribution of the stresses from each species of lengths $L=1,2,3$, or 4 for the $N=1,000$ case.

$1,1.43,1.43$ and 1.43. Note that breakage and reforming is occurring at each time step. That is a chain of $L$ dumbbells may break at one of its nodes becoming two chains of lesser $L$, or may join with another chain to form a chain of more than $L$ dumbbells so that the tagged members of an ensemble of length $L$ change at each time step. For an even longer mean node lifetimes namely $\tau=10$, and the same number of nodes $N=1,000$ (results not shown), the relaxation times of the chains for $L=1,2,3,4$ are respectively $1,1.43,1.43,2$. Thus the relaxation time for longer chains increases (for each mean node lifetime) as one would expect. Note that a two mode exponential is not a good fit to the curve in Fig. 8 (left) indicating that chains with $L>4$ (we have only shown up to 4 in Fig 8 (right)) have even longer ensemble relaxation times.

\section{Steady Shear FLOW: SHEAR THINNING/THICKENING}

The discussions up to now have assumed equilibrium, or close to equilibrium (small amplitude, linear) conditions. In this section we consider larger amplitude deformations, in particular steady shear-rate controlled flow. Four cases are considered in this section:

- In Case I, the mean node lifetime $\tau$ and the capture radius $d$ are both constant, the case which is discussed in equilibrium and step strain in the previous two sections.

- In Case II, the mean lifetime of a node $\tau$ is governed by the force balance between the attractive node and the connected bead-springs (see Equation (5)), while the capture radius $d$ remains constant.

- In Case III, both the mean lifetime $\tau$ and the capture radius $d$ depend on the attached chain stretch through the forces as in the first scenario in Section 3 (see Equations (5) and (6)). 
- Case IV corresponds to the second scenario in Section 3 in which the mean lifetime $\tau$ and the capture radius $d$ are governed by the energy balance (see Equations (7) and (8)).
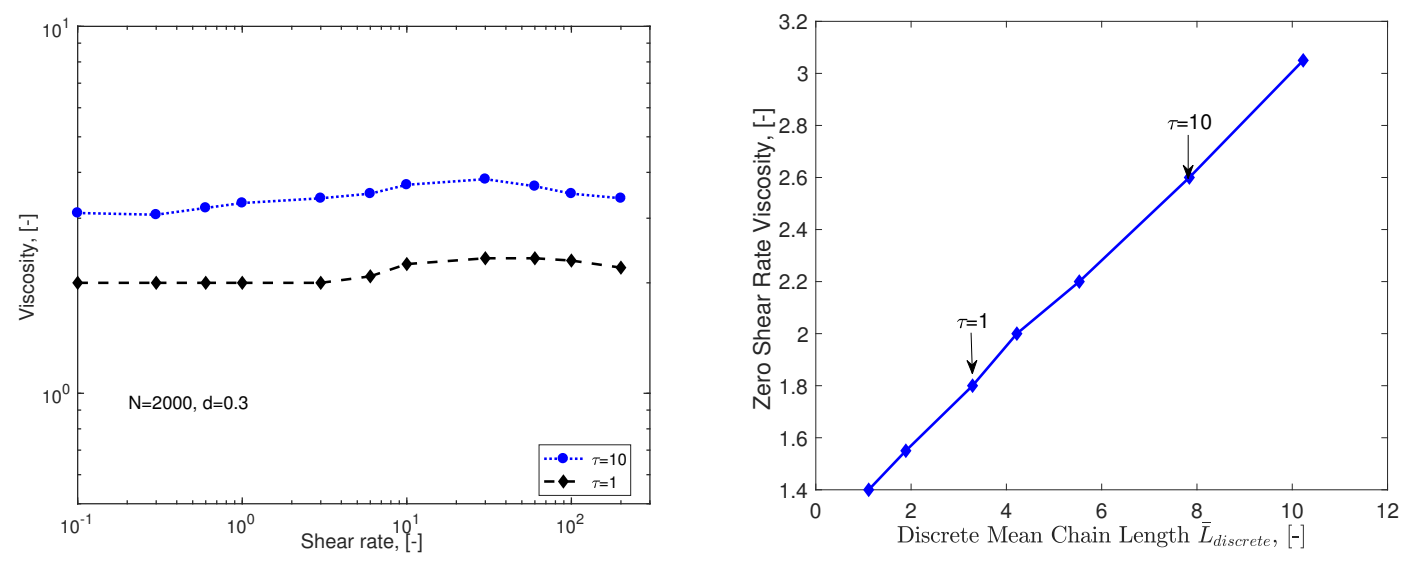

FiguRE 9. The left figure shows the viscosity as a function of the shear rate for Case I. In the simulation, the lifetime $\tau$ is a constant. The attractive distance $d=0.3$. The right figure shows the zero shear rate viscosity as a function of discrete mean chain length. The discrete mean chain length is related to $\tau$ (see Fig. 5). In both figures, $N=2,000$.

Fig. 9 (left) shows the viscosity as a function of shear rate for constant mean node lifetimes of $\tau=1$ and 10 for $N=2000$ and for a constant capture radius $d=0.3$, that is, the result of Case I. The viscosity is constant except between shear rates of roughly 1 and 30 where there is shear thickening. As the papers [20,21, 23] point out the algorithm in [20] allows for shear thickening behavior because under that algorithm the bead on a stretched chain has an enhanced chance of recombining since as the chain relaxes the bead covers more territory than a bead on a lesser stretched chain. In $[21,22,23]$ the equivalent, since they do not track the topology of the network, is to model the attachment rate of their unattached chains as proportional to the stretch of the dumbbell, in particular the attachment rate is taken to be $\alpha_{0}+\alpha_{1} \mathbf{Q}$. Note, that for larger $\tau$, the zero shear rate viscosity is larger. Figure 9 (right) shows the zero shear rate viscosity as a function of the discrete mean chain length $\bar{L}_{\text {discrete }}$ (which is related to the node lifetime as in Fig. 5). The figure shows that this dependence is linear.

In Case II, the capture radius remains constant, but the breakage rate is subjected to the potential well (force) breakage (see Equation (5)). Fig. 10 (left) compares the viscosity as a function of shear rate for Case I and Case II when $\tau=\tau_{0}\left(U_{0}\right)=10$. The curves for the stretch dependent breakage show pronounced shear thinning before the onset of shear 

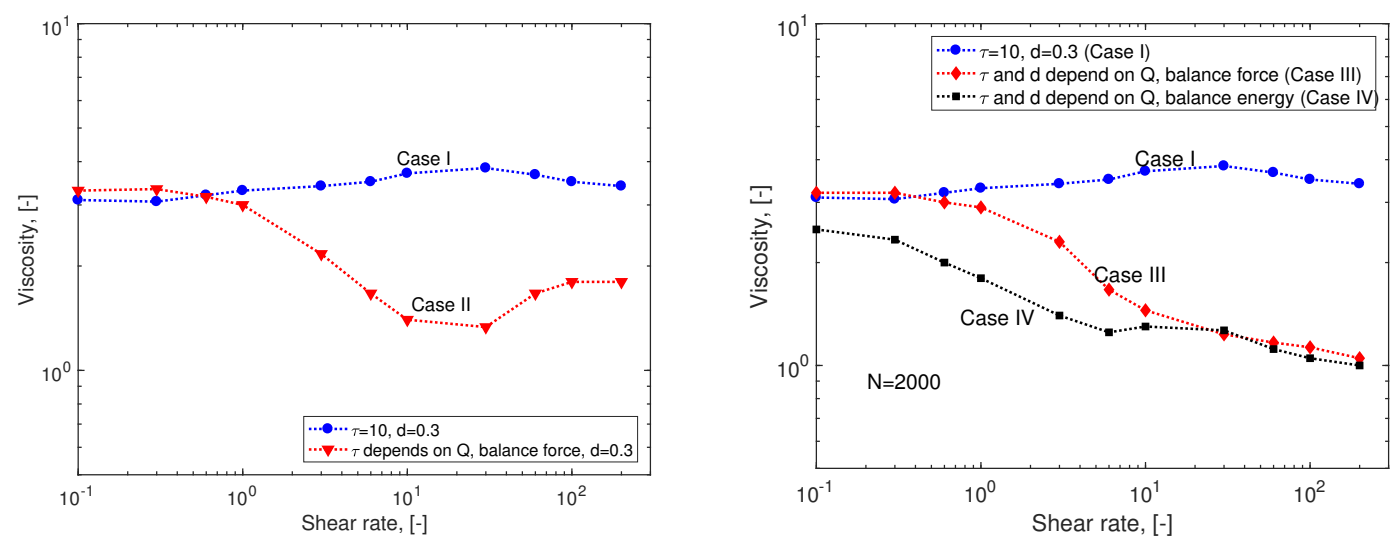

Figure 10. Left figure: Comparison of viscosities of Case I and Case II as functions of shear rates. In Case I, $\tau=10, d=0.3$; in Case II, $d$ remains as a constant 0.3 , but $\tau$ follows Equation (5) and $U_{0}=10, \tau_{0}\left(U_{0}\right)=10$. Right figure: Comparison of viscosities of Case I, Case III (force balance scenario), and Case IV (energy balance scenario) as functions of shear rates. Case I has the same parameter as in the left figure. In Case III and IV, $U_{0}=10, \tau_{0}\left(U_{0}\right)=10$ and $d_{0}=0.3$.

thickening. This shear thinning is anticipated since the mean node lifetime (the inverse of the breakage rate) decreases as the chain stretch increases. Shear thickening takes over after the thinning, beginning at shear rates 30. This shear thickening is due to the constant capture radius $d$ as explained in the previous paragraph. To clarify that the shear thickening is dependent on the reforming kinetics, we plot the viscosity as a function of shear rate for Case III and Case IV in Fig. 10 (right). The red diamond curve (Case III) has the same breakage rate as the red triangle curve in the left figure, but the capture radius $d$ in Case III is consistently set to be $\min \left(d_{0}, d_{F}(Q)\right)$. The shear thickening that appears in the left figure for Case II is no longer present.

For Case IV (Fig. 10 right), in which the breakage rate and recombination depend on $Q$ with the functional forms derived from the energy balance, the mixture also exhibits the shear thinning as the shear rate increases, however, the decrease of viscosity occurs at a smaller shear rate than for the force balance curve (Case III). The latter exhibits a plateau for shear rates less than 1, and the shear thinning doesn't take over until shear rate is roughly 1 . This is because in the force balance case, $\tau$ decreases as $Q$ increases like $e^{-d_{0}^{2} Q^{2} / 4 U_{0}}$. When $Q$ increases from 0 to 5 , in our parameter range, $\tau$ only decreases by $O\left(10^{-2}\right)$ and thus the change has very little effect on the viscosity. For the energy balance case $\tau$ decreases as $Q$ increases like $e^{-d_{0} Q+d_{0}^{2} / 2}$, therefore when $Q$ increases from 0 to 5 , there is a change in $\tau$ of $O\left(10^{-1}\right)$. Note that for a shear rates up to 1 the mean stretch $Q$ stays below 5 (see Fig. 14), and for those values of $Q$ the capture radius $d$ is dominated 
by $d_{0}$.
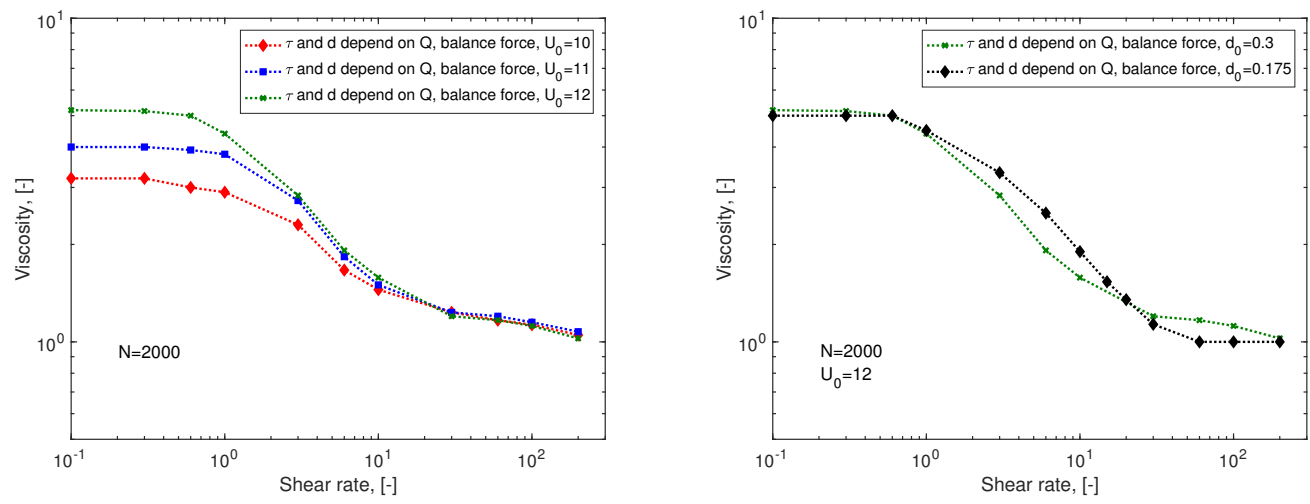

FIgURE 11. Viscosity as a function of shear rate for Case III. The left figure is for various $U_{0}$ for $d_{0}=0.3$, the right figure is for two different values of $d_{0}$ for $U_{0}=12$. In both figures $N=2,000$.

The dependence of the viscosity at a given shear rate on the node attractive energy $U_{0}$ and on the base node attractive radius $d_{0}$ is explored in Fig. 11 for the force balance case (Case III). The left figure shows the viscosity versus shear rate curves for $d_{0}=0.3$ but increasing $U_{0}$. Since $\tau_{0}\left(U_{0}\right) \propto e^{U_{0}}$, as $U_{0}$ increases the mean lifetime at equilibrium increases significantly and the zero shear rate viscosity increases. Furthermore, at shear rates larger than 10 the curves are close to indistinguishable from each other. This is because when the shear rate is larger than 10 , the viscosity is mainly controlled by the reforming mechanism. The capture radius is defined as $d=\min \left(d_{0}, 2 U_{0} / Q\right)$. When $Q$ is less than $2 U_{0} / d_{0}$, which is $20 U_{0} / 3$ for $d_{0}=0.3$, the $\min \left(d_{0}, 2 U_{0} / Q\right)$ equals $d_{0}$. When $Q$ is larger than this number, $\min \left(d_{0}, 2 U_{0} / Q\right)=2 U_{0} / Q$. The change of $U_{0}$ from 10 to 12 produces at most an $O\left(10^{-2}\right)$ change in $d$.

Figure 11 (right) shows the same scenario but with $U_{0}=12$ and varying $d_{0}, d_{0}=$ $0.175,0.3$ respectively. Note that for this force balance scenario the parameter $d_{0}$ occurs both in the node lifetime as $e^{-\frac{d_{0}^{2} Q^{2}}{4 U_{0}}}$ and in the capture radius as $d=\min \left(d_{0}, 2 U_{0} / Q\right)$. For shear rate is less than 1 , this changing $d_{0}$ has little effect on the viscosity plateau because this exponential form is close to 1 as mentioned earlier. When the shear rate is bigger than 1 , the mean node lifetime diverges from the value 1 . For a smaller $d_{0}$ the equivalent breakage rate occurs for a larger $Q$, so larger $\dot{\gamma}$. Therefore the viscosity is higher at a given shear rate $1<\dot{\gamma}<10$ for the smaller $d_{0}$. The capture radii remain constant for $\dot{\gamma}$ roughly less than 10 .

The analogue of Fig. 11 (left) for the energy balance scenario is shown in Fig. 12 for the same $U_{0}$ 's. As in Fig. 11 the zero shear rate viscosity increases with increasing $U_{0}$ and 
the viscosity curves for varying $U_{0}$ are indistinguishable for larger shear rates, in this case above 3 . The steepest portion of these curves is for $U_{0}=12$ at a shear rate of 1 .

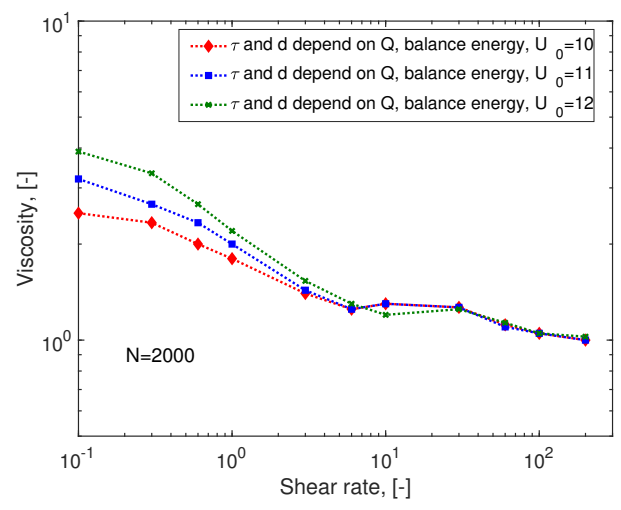

Figure 12. Viscosity as a function of shear rate for Case IV for various $U_{0}$, $d_{0}=0.3 . \quad N=2,000$.

These stretch dependent breakage and reforming scenarios, at least in these parameter ranges, are not sufficient to yield a non-monotone apparent shear-rate control flow curve. A non-monotone flow curve would be of interest to mimic the macroscale wormlike micellar shear banding models' behavior. Other studies [21, 28, 29] have considered FENE springs as opposed to Hookean springs, and [28] considered a FENE force dependent breakage, a much more abrupt cut-off of the chain stretch than our postulated breakage rates. In those studies a parameter range for which the homogeneous flow curve becomes nonmonotonic was found. In our simulations the length of chains as well as their connection configurations change at each time step. This make an implicit scheme as in [21], the optimum scheme for FENE springs, significantly challenging numerically.

It is interesting to investigate the distribution of the stretch of the dumbbells and of the length of the chains at steady state and that dependence on the breaking/reforming functional forms. For Case I, Fig. 13 top row shows the distribution of the number of chains with $L$ dumbbells at steady state after imposition of shear rates of $\dot{\gamma}=0.1,1,10,100$ respectively, the bottom row shows the distribution of the stretch of individual dumbbells at steady state for the same shear rates. For these curves $N=2,000, \tau=10, d=0.3$. Fig. 14 shows the same results but for the force balance scenario, Case III.

In Fig. 13 it can be seen that the mean chain length increases as the shear rate increases, as does the mean stretch. The increase in the number of dumbbells per chain for high shear rates is due to the reforming algorithm as noted by $[21,23]$ in which stretched (longer) chains have a higher probability of attaching to the network than unstretched chains. Fig. 13 bottom shows that dumbbells are more highly stretched as the shear rate increases with 

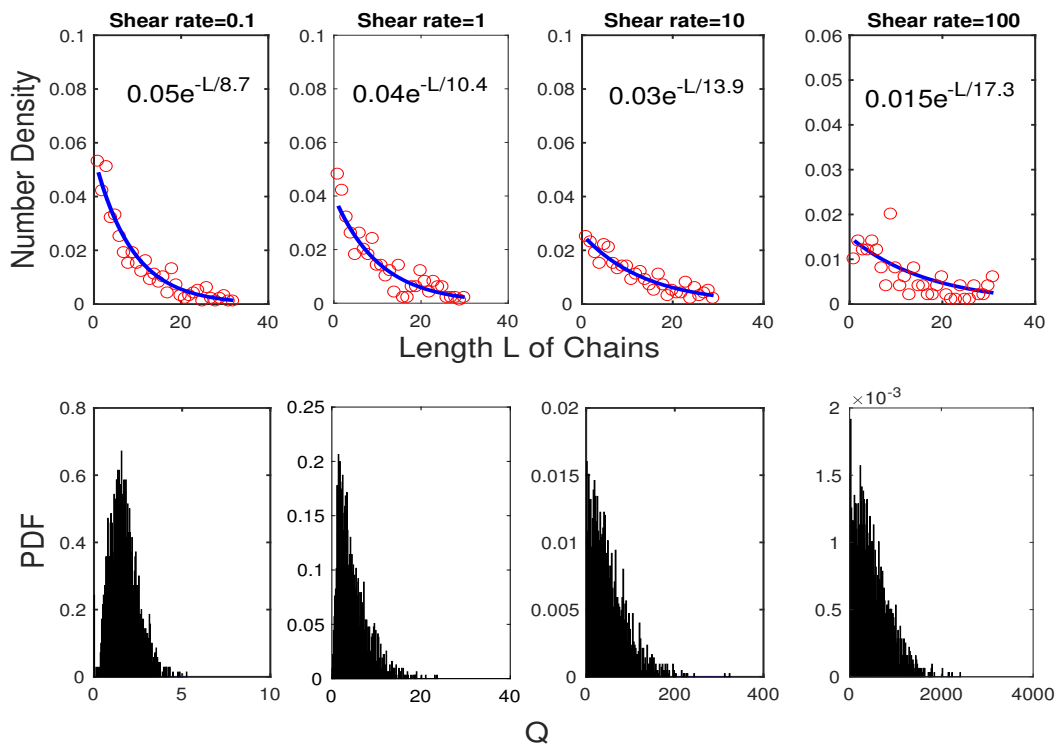

FiguRE 13. Number density of chains containing $L$ dumbbells and the distribution of stretch of the dumbbells for selected shear rates when the mean lifetime of nodes $\tau$ and capture radius $d$ are constant (Case I). Here $N=2,000, \tau=10$, $d=0.3$. This situation corresponds to the blue (solid circle) curve of Fig. 10.

a maximum stretch of roughly 5 for a shear rate of 0.1 , and of 2,000 for a shear rate of 100 . Fig. 14 (top row), in which the mean node lifetime and the capture radius now depend on the local dumbbell stretch according to the force balance (Case III), shows an opposite trend for the distribution of the number of dumbbells per chain. In this case the mean number of dumbbells in a chain decreases as the shear rate increases, with the longest chain containing roughly 35 dumbbells at a shear rate of $0.1,20$ at a shear rate of 10 , and only 8 at a shear rate of 100 . The maximum dumbbell stretch also behaves differently in that its value ranges from 5 for the shear rate of 0.1 (similar to that of Fig. 13) to 1,000 at a shear rate of 100, much less stretched than the dumbbells of Fig. 13. These changes are due to the stretch enhanced breakage as the local shear rate increases.

While this section has dealt with the steady state, we considered a quick look at the time evolution to steady state. Fig. 15 shows the transient behavior of the shear stress and of the first normal stress difference, $N_{1}$, as they evolve to their steady state values. The figures show the results for two selected shear rates $\dot{\gamma}=0.5$ (left figure) and $\dot{\gamma}=10$ (right figure) for the case in which the mean node lifetime $\tau$ and the capture radius $d$ depend on $Q$ as in the force balance (Case III). In these shear rate controlled simulations the shear rate was jumped up to its final value. The corresponding steady state shear stress and viscosity curves were shown in Fig. 11 (left), the green cross curve. The value $\dot{\gamma}=0.5$ 

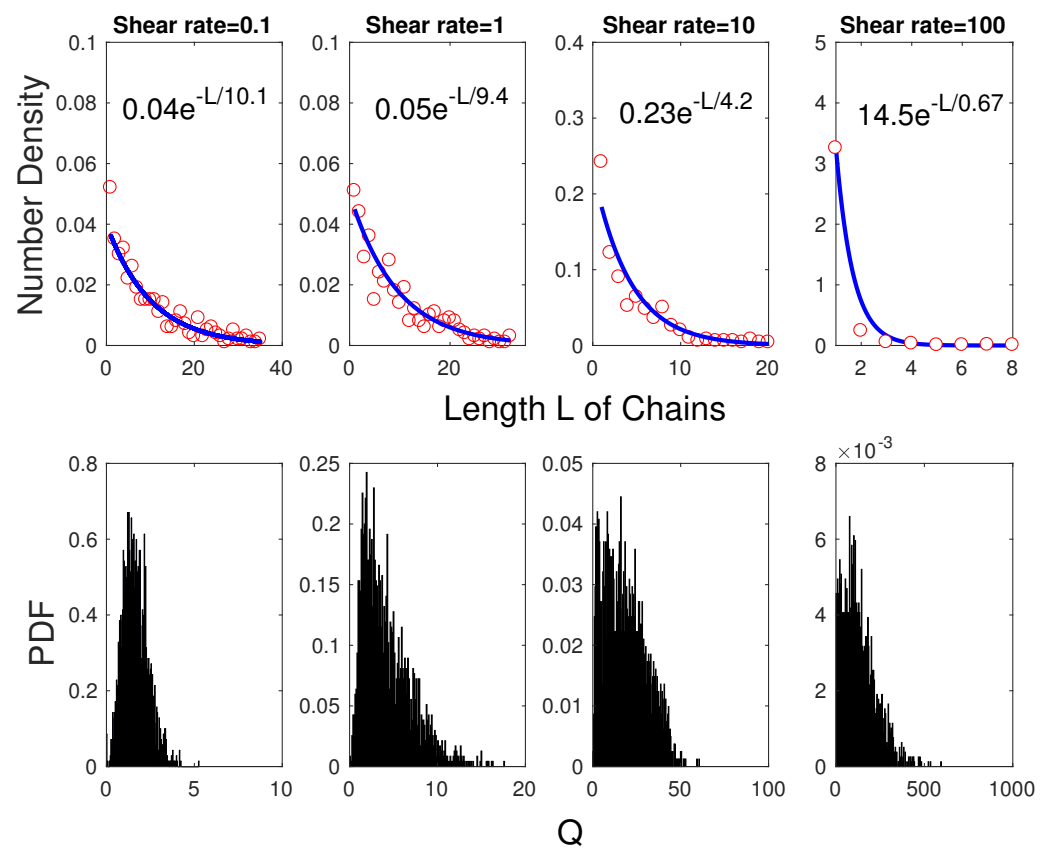

FiguRE 14. Number density of chains containing $L$ dumbbells and the distribution of stretch of the dumbbells for selected shear rates when the lifetime of nodes $\tau$ and the capture radius $d$ depend on $Q$. Here $N=2,000, U_{0}=10, \tau_{0}\left(U_{0}\right)=10$, $d_{0}=0.3$. This situation corresponds to the red (dimond) curve of Fig. 10

was selected as a representative of the regime of shear rates for which the shear stress is linearly increasing with shear rate, that is the viscosity is roughly constant. The value $\dot{\gamma}=10$ was selected as a representative of the regime in which the viscosity decreases as the shear rate increases, that is a representative of the shear thinning portion of the curve. For the value $\dot{\gamma}=0.5$ both the shear stress and the first normal stress difference increase monotonically in time with the shear stress reaching its steady state value earlier than the first normal stress difference. For the value $\dot{\gamma}=10$, both the shear stress and first normal stress curves overshoot, that is first increase and then decrease to their steady state values. This overshoot in the shear stress curve in time has been attributed, by [30], to being an indication of shear banding, at least transient banding. It is to be noted that the overshoot of $N_{1}$ occurs at a later time than the overshoot of the shear stress. This behavior is similar to that shown for the VCM model [9]. Those simulations identified the time of the first normal stress difference overshoot as the same time as the maximum recoil in the formation of the shear banding profile thus attributing that behavior to the elastic effects. 

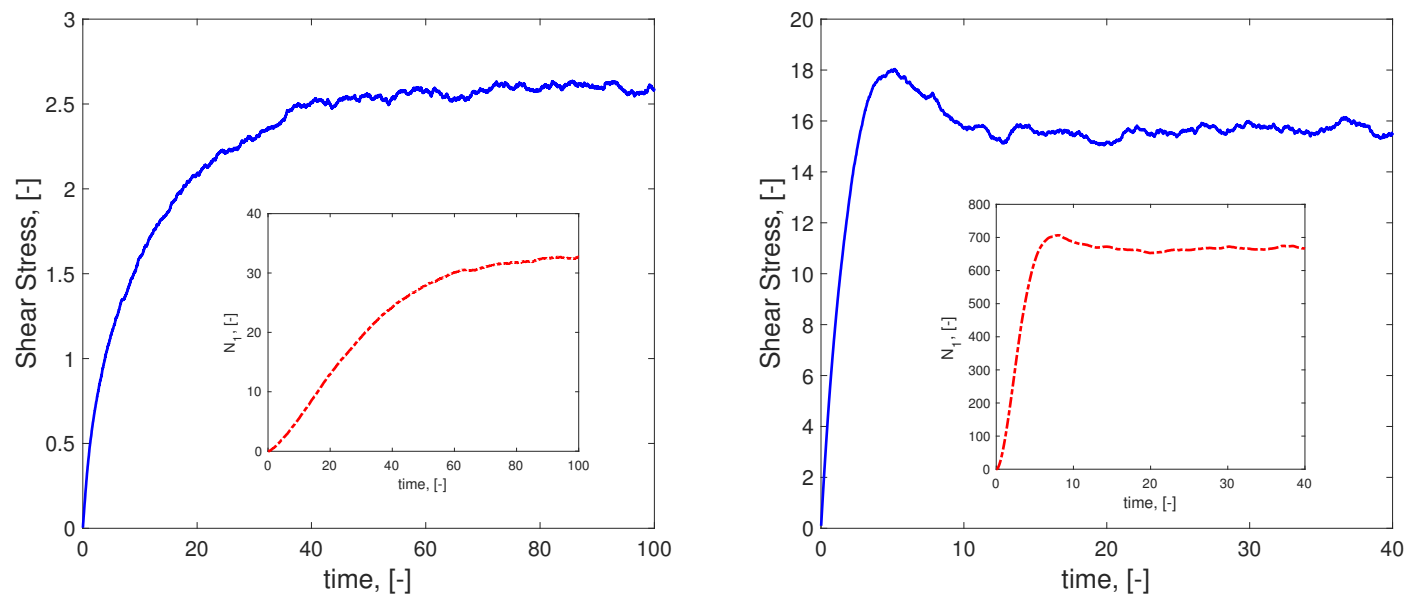

FiguRE 15. The figures show the transient shear stress and first normal stress difference $N_{1}$ for two different $\dot{\gamma}$ 's. The left figure is for $\dot{\gamma}=0.5$ and the right figure is for $\dot{\gamma}=10$. In both figures, $U_{0}=12$, both $\tau$ and capture radius $d$ are dependent on $Q$ with force balance and $d_{0}=0.3, N=2,000$. The curves in both figures are an average of five simulations.

\section{Conclusion}

In this paper a network model for breaking/reforming chains of dumbbells is developed and is analyzed in equilibrium, under step strain relaxation, and in shear-rate controlled homogeneous flow. The work tracks the topology of the network and in this way is similar to [20]; however, the modeling in this paper incorporates explicit representations of the attractive potential between the chain ends. In the two scenario considered, the breakage and reforming are functions of the chain stretch. The stochastic model is formulated and simulated numerically. When the breakage rate and the capture radius for reforming are constant, the equilibrium distribution of the number of chains of $L$ dumbbell units is a decreasing exponential function of $L$. This result agrees with [16] although in that case the breakage and reforming were continuous processes. Relaxation after a step strain, for a mean node breakage time which is short compared to the single dumbbell relaxation time the stress relaxation is singled exponential - despite the polydispersity of the mixture. As the mean node lifetime increases stress relaxation is, after an initial exponential decay, better fit by a stretched exponential. For a constant mean node lifetime and capture radius the steady state flow curve is roughly a constant, but with a small shear thickening region. When the mean node lifetime is a function of the local attached dumbbell stretch, the capture radius still a constant, the shear stress shows strong shear thinning, followed by thickening. When the capture radius is also a function of the local chain stretch, for the range of parameters examined, the shear stress shows strong shear thinning, followed by either no or strongly reduced shear thickening. 


\section{REFERENCES}

[1] R.G. Larson. The Structure and Rheology of Complex Fluids. Oxford University Press, New York, 1999.

[2] J P. Rothstein. Strong flows of viscoelastic wormlike micelle solutions. Rheol. Reviews., pages 1-46, 2008.

[3] J. F. Berret. Rheology of Wormlike Micelles : Equilibrium Properties and Shear Banding Transition. Springer: Dordrecht, 2005.

[4] S.M. Fielding. Complex dynamics of shear banded flows. Soft Matter, 2:1262-1279, 2007.

[5] Y.T. $\mathrm{Hu}$ and A. Lips. Kinetics and mechanism of shear banding in entangled micellar solutions. $J$. Rheol., 49:1101-1027, 2005.

[6] E. Michel, J. Appell, F. Molino, J. Kieffer, and G. Porte. Unstable flow and nonmonotonic flow curves of transient networks. J. Rheol., 45:1465, 2001.

[7] P. A. Vasquez, G. H. McKinley, and L. P. Cook. A network scission model for wormlike micellar solutions I: Model formulation and homogeneous flow predictions. J. Non-Newtonian Fluid Mech., 144:122-139, 2007.

[8] M. Cromer, L.P. Cook, and G.H. McKinley. Extensional flow of wormlike micelles. Chem. Eng. Sci., 64:4588-4596, 2009.

[9] L. Zhou, G. H. McKinley, and L. P. Cook. Wormlike micellar solutions: III. VCM model predictions in steady and transient shear flows. J. Non-Newt. Fluid Mech., 211:70-83, 2014.

[10] N. Germann, L.P. Cook, and A.N. Beris. Nonequilibrium thermodynamic modeling of the structure and rheology of concentrated wormlike micellar solutions. J. Non-Newtonian Fluid Mech., 196:51-57, 2013.

[11] O. Radulescu and P. D. Olmsted. Matched asymptotic solutions for the steady banded flow of the diffusive Johnson-Segalman model in various geometries. J. Non-Newtonian Fluid Mech., 91:143-164, 2000.

[12] M. E. Helgeson, P. A. Vasquez, E. W. Kaler, and N. J. Wagner. Rheology and spatially resolved structure of cetyltrimethylammonium bromide wormlike micelles through the shear banding transition. J. Rheol., 33:727-756, 2009.

[13] N. Germann, A.K. Gurnon, L. Zhou, L.P. Cook, A.N. Beris, and N.J. Wagner. Validation of constitutive modeling of shear banding, threadlike wormlike micellar fluids. J. Rheol., 60:1-17, 2016.

[14] H. Rehage and H. Hoffmann. Viscoelastic surfactant solutions: Model systems for rheological research. Molecular Physics, 74:933-973, 1991.

[15] M. Filali, E. Michel, S. Mora, F. Molino, and G. Porte. Stress relaxation in model transient networks: Percolation and rearrangement of the crosslinks. Colloids and Surfaces A: Physicochemical and Engineering Aspects, 183-185:203-212, 2001.

[16] M.E. Cates. Reptation of living polymers: Dynamics of entangled polymers in the presence of reversible chain-scission reactions. Macromolecules, 20:2289-2296, 1987.

[17] W. Zou and R.G. Larson. A mesoscopic simulation method for predicting the rheology of semi-dilute wormlike micellar solutions. J. Rheol., 58:681-721, 2014.

[18] N. A. Spenley, M. E. Cates, and T. C. B. McLeish. Nonlinear rheology of wormlike micelles. Phys. Rev. Lett., 71:939-942, 1993.

[19] M.E. Cates. Nonlinear viscoelasticity of wormlike micelles (and other reversibly breakable polymers). J. Phys. Chem., 94:371-375, 1990.

[20] B.H.A.A. van den Brule and P.J. Hoogerbrugge. Brownian dynamics simulation of reversible polymeric networks. J. Non-Newtonian Fluid Mech., 60:303-334, 1995.

[21] J.G. Hernandez Cifre, Th.M.A.O.M. Barenbrug, J.D. Schieber, and B.H.A.A van den Brule. Brownian dynamics simulation of reversible polymer networks under shear using a non-interacting dumbbell model. J. Non-Newtonian Fluid Mech., 113:73-96, 2003.

[22] J.G.H. Cifre, R. Pamies, A.L. Kjoniksen, K.D. Knudsen, B. Nystrom, and J.G. de la Torre. Brownian dynamics simulation of reversible polymer networks using a non-interacting bead-and-spring chain model. J. Non-Newtonian Fluid Mech., 146:3-10, 2007. 
[23] A. Vaccaro and G. Marrucci. A model for the nonlinear rheology of associating polymers. J. NonNewtonian Fluid Mech., 92:261-273, 2000.

[24] A. Tripathi, K.C. Tam, and G.H. McKinley. Rheology and dynamics of associative polymers in shear and extension: Theory and experiments. Macromolecules, 39:1981-1999, 2006.

[25] H. C. Öttinger. Stochastic Processes in Polymeric Fluids. Springer-Verlag New York, 1996.

[26] R.B. Bird, C.F. Curtiss, R.C. Armstrong, and O. Hassager. Dynamics of Polymeric Liquids: Vol 2, Kinetic Theory. John Wiley and Sons, New York, second edition, 1987.

[27] A.W. Lees and S.F. Edwards. The computer study of transport provessess under extreme conditions. J.Phys.C: Solid State Phys., 5:1921-1929, 1972.

[28] M.K. Sing, Z-G. Wang, G.H. McKinley, and B.D. Olsen. Celebrating Soft Matter's 10th anniversary: Chain configuration and rate-dependent mechanical properties in transient networks. Soft Matter, 11:2085-2096, 2015.

[29] E.B. Stukalin, L-H. Cai, N.A. Kumar, L. Leibler, and M. Rubinstein. Self-healing of unentangled polymer networks with reversible bonds. Macromolecules., 46:7525-7541, 2013.

[30] S.M. Fielding. Triggers and signatures of shear banding in steady and time-dependent flows. J. Rheol., 60:821-834, 2016. 